\title{
Algunas de las aplicabilidades actuales de las investigaciones en neologia y sobre los neologismos
}

\author{
MaRía TADEA Díaz Hormigo \\ Catedrática de Lingüística General \\ Facultad de Filosofia y Letras \\ Departamento de Filología e Instituto Univer- \\ sitario de Investigación en Lingüística Apli- \\ cada \\ Avda. Gómez Ulla, s/n ${ }^{\circ}$ \\ 11003 Cádiz \\ E-mail: tadea.diaz@uca.es
}

\author{
ÉRIKA Vega MoReno \\ Profesora sustituta interina de Lingüística \\ General \\ Facultad de Filosofia y Letras \\ Departamento de Filología e Instituto Univer- \\ sitario de Investigación en Lingüística Apli- \\ cada \\ Avda. Gómez Ulla, s/nº \\ 11003 Cádiz \\ E-mail: erika.vega@uca.es
}

\begin{abstract}
ALGUNAS DE LAS APLICABILIDADES ACTUALES DE LAS INVESTIGACIONES EN NEOLOGÍA Y SOBRE LOS NEOLOGISMOS
\end{abstract}

RESUMEN: En este artículo mostramos la transferibilidad a otras disciplinas, técnicas y metodologias de los resultados de las investigaciones realizadas sobre un corpus de nuevas unidades léxicas no codificadas lexicográficamente neologismos-. En concreto, describimos las aplicabilidades del estudio de los neologismos detectadas por nuestra parte en los ámbitos de la lexicografia, la política y planificación lingüisticas, la gramática, la terminología, la traductología, la comunicación y asesoramiento lingüisticos, la psicolingüística, la teoria y metodologia de la enseñanza de lenguas, y la lingüistica clínica.

PALABRAS CLAVES: neología; neologismo; lingüística aplicada; aplicabilidades de la lingüística; transferencia del conocimiento.

SUMARIO: 1. Introducción: Conceptos teóricos y criterios metodológicos. 2. Neologia, Lexicografia y Política y planificación lingüisticas. 3. Neologia y Gramática. 4. Neologia y Terminología. 5. Neologia y Traductología. 6. Neología y Comunicación y asesoramiento lingüisticos. 7. Neología, Psicolingüística, Teoria y metodologia de enseñanza de lenguas y Lingüística Clínica. 8. Conclusiones.

\section{SOME OF THE CURRENT APPLICATIONS OF RE- SEARCH IN NEOLOGY AND ON NEOLOGISMS}

ABSTRACT: In this article we will demonstrate the transferability to other disciplines, techniques and methodologies of the results of research carried out on a corpus of lexicographically uncodified lexical units -neologisms-. more specifically, we will describe the applicability of the study of neologisms that we have detected in the fields of lexicography, linguistic policy and planning, grammar, terminology, traductology, linguistic communication and assessment, psycholinguistics, language teaching theory and methodology and clinical linguistics.

KEY WORDS: neology; neologism; applied linguistics; applications of linguistics; knowledge transfer.

SUMMARY: 1. Introduction Theoretical concepts and methodological criteria. 2. Neology, Lexicography and Linguistic policy and planning. 3. Neology and Grammar. 4. Neology and Terminology. 5. Neology and Traductology. 6. Neology and Linguistic communication and assessment. 7. Neology, Psicholinguistics, Language teaching theory and methodology, and Clinical Linguistcs.8. Conclusions.

\section{QUELQUES-UNES DES APPLICATIONS ACTUELLES DE LA RECHERCHE EN NEOLOGIE ET SUR LES NEOLOGISMES}

RÉSUMÉ: Dans cet article, nous montrons la transférabilité vers d'autres disciplines, techniques et méthodologies des résultats des recherches effectuées sur un corpus de nouvelles unités lexicales non codées lexicographiquement - les néologismesPlus précisément, nous décrivons l'applicabilité de l'étude des néologismes que nous avons détectés dans les domaines de la lexicographie, de la politique et de la planification linguistiques, de la grammaire, de la terminologie, de la translatologie, de la communication linguistique et conseils linguistiques, de la psycholinguistique, de la théorie et de la méthodologie de l'enseignement des langues, et de la linguistique clinique.

MOTS CLÉS: néologie; néologisme; linguistique appliquée; applications de la linguistique; transfert de connaissances.

SOMMAIRE: 1. Introduction Concepts théoriques et critères méthodologiques. 2. Néologie, lexicographie et politique et planification linguistiques. 3. Néologie et grammaire. 4. Néologie et terminologie. 5. Néologie et traductologie. 6. Néologie et communication linguistique et conseils linguistiques. 7. Néologie, psicholinguistique, théorie et méthodologie de l'enseignement des langues, et linguistique clinique. 8 . Conclusions.
$27 / 07 / 2018$

$31 / 09 / 2018$

$08 / 11 / 2018$

$01 / 12 / 2018$ 


\title{
Algunas de las aplicabilidades actuales de las investigaciones en neologia y sobre los neologismos ${ }^{1}$
}

\author{
María TADEA Díaz Hormigo \& ÉRIKA Vega Moreno
}

\section{INTRODUCCIÓN: CONCEPTOS TEÓRICOS Y CRITERIOS METODOLÓGICOS}

Como explicitamos, sobre todo, en M. T. Díaz Hormigo (2008) y É. Vega Moreno (2010), existe un amplio consenso entre los autores más representativos en la materia para entender por neologia tanto el proceso de creación de nuevas unidades léxicas como la disciplina que estudia todo lo relacionado con este proceso de renovación y actualización del léxico de una lengua. Asimismo, comúnmente, se suele llamar neologismo al producto que resulta del proceso referido, esto es, la nueva unidad léxica, que puede surgir o bien por una necesidad del hablante de denominar un nuevo concepto, objeto o realidad -la neología denominativa o referencial- o bien por una necesidad del hablante de introducir matices subjetivos o nuevas formas expresivas en la comunicación -la neología estilística o expresiva-. En cualquier caso, la nueva unidad léxica puede ser de creación reciente solo en su significante (por ejemplo ${ }^{2}$, nini, mani, manifa, infoxicación, infopista) o en su significante y en su significado (por ejemplo, conspiranoico, -ca; enoturismo, aporofobia, tacticismo, semana blanca, oscarizar) -la neología formal, ordinaria, de forma o de forma y sentido-, solo en su significado (por ejemplo, clonar, altavoz, tentáculos, nicho, técnico, salir del armario) -la neología semántica o de sentido-, o bien tratarse de una unidad léxica recientemente incorporada de otra lengua (por ejemplo, post, biopic, phubbing, spoiler, risotto, reiki, wok, feng shui, savoir faire, femme fatale) - la neología de préstamo ${ }^{3}$. . Ahora bien, estas nuevas unidades léxicas, que, por haber sido in-

\footnotetext{
1 Nuestra indagación se inserta en la línea de investigación en "Neología" del "Gabinete de Industrias de la Lengua" del "Instituto Universitario de Investigación en Lingüística Aplicada" de la Universidad de Cádiz. Esta línea es desarrollada, bajo la dirección de la Profa. Dra. María Tadea Díaz Hormigo, por miembros del grupo de excelencia del Plan Andaluz de Investigación, Desarrollo e Innovación "Semaínein” (HUM 147), en el marco del nodo NEOUCA, integrado en la red de observatorios NEOROC (Red de Observatorios de Neología del Castellano), coordinada por el Observatori de Neologia de la Universitat Pompeu Fabra, mediante convenio especifico de colaboración entre la Universidad de Cádiz y la Universitat Pompeu Fabra para la participación en la red NEOROC y la cooperación en investigación en neología (cf. http://www.iula.upf.edu/rec/neoroc). Asimismo, el trabajo que presentamos se enmarca en el proyecto "Comunicación especializada y terminografia: usos terminológicos relacionados con los contenidos y perspectivas actuales de la semántica léxica" (FFI2014-54609-P) (Calificación: A), dirigido por el Prof. Dr. Miguel Casas Gómez y financiado por el Ministerio de Economía y Competitividad (Programa Estatal de Fomento de la Investigación Científica y Técnica de Excelencia. Subprograma Estatal de Generación del Conocimiento).

${ }^{2}$ Los ejemplos de este párrafo han sido tomados de http://blogscvc.cervantes.es/martesneologico, donde, además, se da una explicación para estas unidades consideradas neológicas. 3 A la que también se asimila la neología por generalización de palabras, esto es, la determinación de las palabras de una determinada diatopía, diastratía o diafasía que pasan a
} 
corporadas recientemente al acervo léxico de los hablantes, no aparecen registradas en los que se consideran diccionarios más representativos de la lengua ni, por tanto, han sido sometidas al proceso -normativo, institucional y externo- de normalización, deben presentar cierta difusión en el uso para poder ser denominadas, con toda propiedad, neologismos.

En este artículo, nos basamos en conclusiones derivadas de las investigaciones efectuadas sobre los resultados de los análisis de nuevas unidades léxicas de la lengua común, candidatas a neologismos o ya calificables como tales, documentadas en a) textos orales y textos escritos de medios de comunicación y textos escritos de publicaciones diversas dirigidas a un público general, recogidos manualmente de forma más rigurosa sobre todo desde 2006; b) textos de prensa escrita vaciados por el Banco de datos del Observatori de Neologia (BOBNEO) del Institut de Lingüística Aplicada (IULA) de la Universitat Pompeu Fabra (http: / / obneo.iula.upf.edu/bobneo), que incluye las nuevas unidades léxicas detectadas en la prensa de amplia difusión de España y países de América Latina, sirviéndonos de las documentadas a partir de 2008; c) textos publicitarios orales y escritos del lenguaje de la publicidad en general, desde 2008, y de los sectores de la estética y la peluquería en particular, desde 2009, extraídos de revistas de carácter divulgativo, anuncios de televisión, carteles y vallas publicitarias, envases y webs de las empresas vendedoras, y d) el blog "Martes neológico", coordinado por Observatori de Neologia del IULA y el Instituto Cervantes por medio de su Centro Virtual (cf. http:/ / blogscvc.cervantes.es/martes-neologico). La metodología de trabajo empleada en el análisis ha sido la diseñada por el IULA (cf. M. T. Cabré et al. 2004), modificada, en lo que se refiere a los criterios para el reconocimiento de neologismos por É. Vega Moreno (2016a, 2017c y en prensa) y É. Vega Moreno y E. Llopart Saumell (2017) ${ }^{4}$.

Pretendemos dar cuenta de que las indagaciones realizadas por nuestra parte ${ }^{5}$ arrojan unos resultados que contribuyen a mostrar la interrelación e

\footnotetext{
ser palabras de la lengua común o general o de arcaísmos que revitalizan su uso en la lengua común o general. Sirvan como ejemplos las voces de amplio uso y difusión en nuestra lengua escarchar, registrada, aunque como de Argentina y Uruguay, ya en el Diccionario de la lengua española (2014, 23 ${ }^{\text {a }}$ ed.), y soplar 'beber o comer mucho', jalar 'comer con mucho apetito', sobar 'dormir', viejo, - a 'padre o madre de una persona' y pedo 'borrachera', todas, aunque con la marca 'coloquial', sí registradas en el Diccionario de la lengua española (2014, $23^{\mathrm{a}}$ ed.).

${ }^{4} \mathrm{En}$ efecto, aunque tomando como base los criterios y parámetros propuestos y aceptados comúnmente por los neólogos para la determinación del carácter neológico de una unidad léxica: la diacronia, la lexicografia, la inestabilidad sistemática y la psicología (cf. M. T. Cabré 1993: 445); en É. Vega Moreno (2016a, 2017c y en prensa) y É. Vega Moreno y É. Llopart Saumell (2017) se propone distinguir entre a) criterios de obligado cumplimiento: el criterio psicológico o de sentimiento de neologicidad; el criterio del filtro lexicográfico, y el criterio de la difusión en el uso, para constatar que no se trata de un neologismo efimero, pues debe haber sido documentado en al menos diez contextos diferentes, y b) criterios de no obligado cumplimiento: el criterio diacrónico, o de aparición reciente de un candidato a neologismo, y el criterio de la inestabilidad sistemática, pues el candidato a neologismo puede presentar o no signos de inestabilidad formal y/o semántica.

${ }^{5}$ Para un resumen y reseña de las mismas, cf. específicamente M. T. Díaz Hormigo, C. Varo Varo y É. Vega Moreno (2016).
} 
interdependencia de la neología y otros ámbitos de la lingüística aplicada, aunque con mayor evidencia, al menos, entre la neología y los campos aplicados de la lexicografia, la politica y planificación lingüísticas, la gramática, la terminología, la traductología, la comunicación y el asesoramiento lingüísticos la psicolingüística, la teoría y la metodología de la enseñanza de lenguas, y la lingüística clínica, por lo que resulta procedente destacar la transferibilidad y las aplicabilidades actuales de los resultados de las investigaciones en neología hacia/desde estos otros sectores de la lingüística aplicada.

\section{NeOLOGÍA, LEXICOGRAFÍA Y POLÍtica Y PLANIFICACIÓN LINGÜíSTICAS}

No cabe duda de que una de las manifestaciones más visibles y útiles de las aplicaciones de las investigaciones sobre neologismos es su contribución a la actualización de los diccionarios de lengua general con la inclusión de nuevas unidades léxicas, siendo este -contribuir a la actualización del léxico de los diccionarios de lengua general- uno de los objetivos que se proponen cubrir con mayor ahínco los distintos proyectos sobre neología y neologismos que se desarrollan en la actualidad ${ }^{6}$.

Ahora bien, aunque por lo general parece comúnmente aceptado que las unidades lingüísticas que deben ser registradas en los diccionarios generales deben ser únicamente las unidades léxicas, suele ser práctica habitual la inclusión, como artículos independientes, en la macroestructura de estos diccionarios, además de las denominadas palabras gramaticales, también de los elementos que intervienen en la formación de palabras, esto es, prefijos, sufijos y raíces cultas de origen griego o latino. Se justifica la consignación de estos elementos formativos por la imposibilidad de registrar como entradas del diccionario todos los resultados y las creaciones léxicas pertinentes, que dan cuenta del caudal no finito de palabras posibles en una lengua según las reglas de formación de palabras, y por el evidente ahorro de espacio al economizar el número de entradas de la obra lexicográfica. En este sentido, nuestras investigaciones sobre las nuevas creaciones léxicas por derivación (cf. M. T. Díaz Hormigo, 2011a; 2011b; 2012a; M. T. Díaz Hormigo y C. Varo Varo, 2012) han revelado que los formantes de estas nue-

\footnotetext{
${ }^{6}$ Sirvan como ejemplo los objetivos perseguidos por la Red de Observatorios de Neología del Castellano (NEOROC), coordinada por el Observatori de Neologia de la Universitat Pompeu Fabra, a la que hicimos referencia en la nota introductoria de este trabajo. En efecto, aunque la finalidad principal de NEOROC ha sido, desde su creación en 2004, la detección, la selección, el análisis, el almacenamiento, la difusión y el estudio contrastivo de la neología léxica de las distintas variedades del español peninsular, para la consecución de este propósito general, este proyecto coordinado (cf. M. T. Cabré et al. 2002: 167) pretende cubrir también los objetivos de 1) constituir un corpus de neologismos de las distintas variedades del español, generado a partir de los medios de comunicación; 2) describir y analizar contrastivamente los recursos del español para actualizar su léxico y poder, también, medir la vitalidad de la lengua; 3) difundir periódicamente las nuevas creaciones léxicas del español, y 4) como ya hemos indicado, contribuir a la actualización del léxico contenido en los diccionarios de lengua general.
} 
vas unidades léxicas, o neologismos, no siempre se ajustan a las informaciones de diversa indole, aunque sobre todo a las de carácter morfológico derivativo, morfofonológico y semántico, consignadas respecto a ellos en los diccionarios generales, pues puede suceder, y de hecho sucede, que estos formantes hayan modificado sus características gramaticales o adquirido acepciones no consignadas en la información que figura en la entrada de los mismos en las obras lexicográficas ${ }^{7}$. Es por ello que entendemos que, tal como desarrollamos en M. T. Díaz Hormigo (2015 y 2017a), los resultados de los análisis de los neologismos formados por derivación pueden contribuir a la actualización de entradas de sufijos, prefijos, circunfijos, elementos compositivos (formantes, en general), de los diccionarios generales, fundamentalmente, en lo que se refiere a inclusión de nuevas acepciones para significantes ya registrados.

Asimismo, el establecimiento de las bases teóricas para la elaboración de diccionarios y glosarios de neologismos y la propia y específica confección de estos deben tener en cuenta los avances en las técnicas de elaboración de diccionarios diseñadas por la lexicografia. De este modo, quedará patente la necesidad de incluir, en las correspondientes entradas lexicográficas, informaciones fonéticas, fonológicas, ortográficas, morfofonológicas, morfológicas, semánticas, sintácticas, y también, en algunos lemas, pragmáticas, estilísticas, sociolingüísticas (variación lingüística diatópica, diastrática, diafásica, diacrónica) y psicolingüísticas, pues todas ellas son necesarias y pueden contribuir a la correcta formulación de la entrada y definición de los neologismos ${ }^{8}$.

\footnotetext{
${ }^{7}$ Sirvan como ejemplo las informaciones consignadas en el Diccionario de la lengua española (2014, $23^{\mathrm{a}}$ ed.) para el formante mega- (1. elem. compos. Significa 'grande'. Megalito, megaterio; 2. elem. compos. Significa 'un millón $\left(10^{6}\right)$ de veces'. Con nombres de unidades de medida, forma el múltiplo correspondiente. (Símb. M); 3. elem. compos. Inform. Significa 'aproximadamente un millón $\left(2^{20}\right)$ de veces'. (Símb. M). Un megabyte), difícilmente aplicables a las creaciones léxicas que figuran en los siguientes contextos extraídos del BOBNEO: "países megadiversos", "la megavendida pulsera", "una colega igualmente megapija", "piel megahidratada", "rendimiento megaespectacular", en los que presenta la noción equivalente a 'muy, grado alto de la propiedad indicada'.

8 En este sentido, podemos hacer referencia al Diccionario de neologismos on line (http://obneo.iula.upf.edu/spes), elaborado por el Observatori de Neologia del IULA, que recoge más de 4000 neologismos en español documentados entre 1989 y 2007, mayoritariamente en prensa escrita, con incorporación, en su última actualización, de los datos procedentes de las variantes del castellano que integran el proyecto Antenas Neológicas. En esta obra lexicográfica, las informaciones que se indican para cada neologismo son el lema, la categoría gramatical, las variantes ortográficas, las variantes morfológicas, la definición, contexto(s) de uso, con indicación de la fuente y fecha de procedencia, variantes sinonímicas, etimología, pronunciación, uso y su aparición en otras obras lexicográficas si la hubiera. Por su parte, en el reciente Diccionario de neologismos del español actual (cf. M. C. Sánchez Manzanares (dir.) et al., 2016) (https://www.um.es/neologismos), que incluye las voces neológicas del español detectadas en la prensa de Murcia y Alicante y las voces usadas en estas provincias de forma diferente respecto a otras variedades del español, se consigna para cada neologismo el lema, variantes que figuren en el propio diccionario, categoría y subcategoría gramatical, la marca diatópica, la marca temática, la definición, el tipo (neologismo formal, préstamo, neologismo semántico, neologismo sintáctico), con especificación del procedimiento de formación; información adicional,
} 
No obstante, tanto para la contribución a la actualización del léxico de los diccionarios de lengua general con la inclusión de nuevas unidades léxicas o neologismos como para la actualización de las entradas de prefijos, sufijos, elementos compositivos y formantes que figuren en estos diccionarios, se ha de atender a la actividad institucional organizada, por los organismos oficiales, de recopilación, registro, difusión e implantación de las nuevas unidades neológicas en el marco general de la política de normalización de una lengua y de su codificación, lo que se observa con particular y contundente evidencia para el proceso de lexicalización y registro en los diccionarios de las unidades léxicas neológicas que presentan inestabilidad formal y/o semántica ${ }^{9}$. Asimismo, los resultados de las investigaciones sobre neologismos pueden contribuir a la elaboración de materiales que faciliten la labor de normalización de los neologismos, atendiendo a la inestabilidad formal y/o semántica que pueden presentar estas nuevas unidades léxicas aún no codificadas.

\section{NeOlogía y Gramática}

Nuestras investigaciones sobre los neologismos que responden a alguno de los procedimientos de la denominada tradicionalmente formación de palabras (cf. M. T. Díaz Hormigo, 2011a; 2011b; 2012a; 2012b; M. T. Díaz Hormigo y C. Varo Varo, 2012) han mostrado que la detección y análisis de las nuevas creaciones léxicas por derivación, composición o parasíntesis sirven también, además de para cubrir los objetivos pretendidos por los proyectos de investigación sobre neologismos en curso (cf. n. 5) y a las finalidades a las que hemos aludido en el apartado 4. de este artículo, para contribuir a la actualización de los presupuestos teóricos sobre formación de pa-

si se considera pertinente; registro en otros diccionarios, si aparece, y contexto(s), con indicación de fuente y fecha.

${ }^{9}$ En efecto, en M. T. Díaz Hormigo (2017a), a propósito de nueva reivindicación de una neología morfológica, dimos cuenta de la problemática de la adscripción en español de las unidades léxicas tomadas o adaptadas recientemente de otras lenguas -neología de préstamo- a un género gramatical o normativo, a un número o a una conjugación verbal, fundamentalmente, por la variabilidad de usos que se observan en las manifestaciones de estas unidades léxicas y las divergencias que presentan estas flexiones, en la mayoría de los casos, respecto a las reglas gramaticales que conforman la norma de la lengua española. Así, examinando las entradas de algunos préstamos del inglés en el BOBNEO (cf. http://obneo.iula.upf.edu/bobneo), observamos a) que el género es masculino en todos las ocurrencias documentadas del sustantivo spoiler, femenino en las de master class (masterclass y máster class) e it girl (itgirl), pero que selfi, selfie en unos contextos aparece con género masculino y en otros con género femenino; b) respecto al número, que en plural es flexionado como en inglés: spoilers; que master class (masterclass y máster class) es invariable en cuanto al número; it girl (itgirl) aparece invariable o bien flexionado: it girl, it girls, y selfi, selfie es flexionado como selfis, selfies, respectivamente, y c) en relación con los verbos tomados del inglés, se procede por lo general a la adición de la desinencia verbal del infinitivo, casi siempre de la primera conjugación (aprochar, clickar y customizar), pero también se han documentados casos de verbos con la adición además de un sufijo derivativo: hackear y renderizar. 
labras contenidos en gramáticas, monografias, artículos, tratados específicos y diccionarios de lingüística ${ }^{10}$ y a la de las informaciones que, sobre sufijos, prefijos, circunfijos, elementos compositivos (formantes, en general), figuran en gramáticas y estudios monográficos específicos de formación de palabras, así como para determinar las tendencias predominantes en los procesos de formación de nuevas unidades léxicas, las dimensiones de los mecanismos de la formación de palabras, la productividad de las realizaciones de un modelo determinado de esquema o estructura de derivación y composición y el grado de productividad de cada formante afijo, su vitalidad y pervivencia.

Como es sabido, las teorías y los presupuestos de las gramáticas y los artículos, monografias y tratados de formación de palabras se basan para sus descripciones en el léxico ya establecido y codificado en los diccionarios, atendiendo a los procesos de formación de palabras y a los patrones morfológicos en los que se basa este léxico existente, sin advertir que esta consideración diacrónica se contrapone al aspecto sincrónico creativo de la competencia léxica, de la creatividad lingüistica o actividad neológica, que es, en definitiva, lo que pretenden explicitar.

En efecto, los resultados de la investigación de nuevas unidades léxicas revelan que estas pueden ser resultado de divergencias de las normas, pautas y reglas de formación de palabras teóricamente establecidas, de tal manera que estas recientes creaciones pueden haber surgido por el empleo de nuevas normas, pautas, reglas; por una modificación de las ya existentes, o bien porque se está transgrediendo la literalidad de una regla necesaria ${ }^{11}$. No obstante, en cualquier caso, habría que decidir si todas las divergencias deben incorporarse, y de qué modo, a las gramáticas, monografias, artículos y tratados específicos de formación de palabras, diccionarios de lingüística, etc.

\footnotetext{
${ }^{10}$ Igualmente, si atendemos a la creación de nuevas unidades léxicas por el procedimiento formal del acortamiento, observamos, frente a lo que se suele indicar al respecto en las fuentes bibliográficas pertinentes, que actualmente los acortamientos pueden efectuarse sobre sintagmas o construcciones (finde, porfa, porsi, sinpa); que empiezan a ser frecuentes los acortamientos por aféresis (kete, de paquete; scope, de cinemascope) y que hay tendencia a la creación de acortamientos monosílabos (pro, de progresista; na, de nada) y trisílabos (manifa, anfeta). Asimismo, respecto a la creación de palabras por siglación, observamos la tendencia a la representación explícita y completa de las siglas en la escritura (emepetrés, gepeese, oenegé, onegé), indicando un estadio más en el proceso de lexicalización de las mismas.

${ }^{11}$ Así, por ejemplo, frente a las reglas, normas y pautas consignadas sobre la flexión de los compuestos sintagmáticos, hemos documentado creaciones neológicas de adjetivo y adjetivo con morfemas de género y número en ambos adjetivos ("una violencia política-étnica", "fines publicitarios-intimidatorios"); de sustantivo y sustantivo coordinados y apositivos que designan entidad animada cuyos sustantivos formantes presentan distinto género $\mathrm{y} / \mathrm{o}$ distinto número (lector-hembra, peces cebra); de sustantivo y sustantivo apositivos que denotan entidad no animada con marca de pluralidad solo en el primer constituyente (carriles-bici, conciertosespectáculo), y de sustantivo y sustantivo que denotan entidad animada o no animada con marca de plural solo en el segundo constituyente ("relación calidad-prestaciones", "la colección de libro-cómics", "los progre-pijos del PSOE"), posibilidades estas a las que no se atiende en los tratados de formación de palabras consultados.
} 


\section{NeOlogía y Terminología}

Son cuatro los sectores en los que se observa con particular rigor la interrelación neología y terminología: 1) en la creación o adaptación de términos para denominar conceptos nuevos de ámbitos científicos y técnicos y de especialidad; 2) en la identificación de los sectores especializados nuevos o recientes; 3) en la simplificación de confluencias de formas designativas para un concepto, y 4) en el hallazgo de lagunas denominativas. Precisamente de la conexión evidente entre neologia y terminología resulta la distinción propuesta por G. Rondeau (1984: 122-124), a partir de la relación de pertenencia, o no, de las nuevas creaciones léxicas al sistema de la lengua y a su diferente ámbito de uso, entre a) la neología léxica de la lengua común (la neologia general o neología propiamente dicha) y b) la neología léxica de las lenguas de especialidad (la neología especializada, terminológica o neonimia).

No obstante, ciñéndonos al ámbito de la neología de la lengua común o general, en relación con la confluencia neología y terminología, arrojan resultados de especial interés analizar los fenómenos de 1) la desterminologización o banalización de términos, esto es, la determinación de los términos de una determinada lengua de especialidad que pasan a ser también palabras de la lengua común o general ${ }^{12}$, y 2) la terminologización de palabras, esto es, la determinación de las palabras de la lengua común o general que pasan a ser también términos de una determinada lengua de especialidad ${ }^{13}$.

\section{NeOLOGía Y TRADUCTOLOGÍA}

La traducción de nuevas unidades léxicas y neologismos es considerada como uno de los mayores retos a los que se enfrentan los traductores, pues, por una parte, deben descifrar, interpretar y resolver el sentido y la función de la nueva unidad léxica de la lengua de partida, y, por otra, hallar el equivalente léxico -si es posible, el difundido en el uso- en la lengua meta o lengua de llegada. Ahora bien, cuando el traductor desconoce -pues puede que no exista- tal equivalente, debe recurrir a la utilización de las estrategias, mecanismos y procedimientos teóricos identificados, precisamente, para paliar y, si es posible, resolver las dificultades que se les presentan en las distintas etapas del proceso de traducción y, entre otras, facilitarles la

\footnotetext{
12 Como son las voces, ya registradas en el Diccionario de la lengua española (2014, 23ª ed.), paracetamol y angioma (del ámbito de la medicina), byte (del ámbito de la informática), célula madre (del ámbito de la biología) o agujero negro (del ámbito de la astronomía), si bien no se encuentra aún en el diccionario académico la voz ibuprofeno a pesar de su extendido uso en la lengua común.

$13 \mathrm{Cf}$. en el Diccionario de la lengua española $\left(2014,23^{\mathrm{a}}\right.$ ed.) las acepciones de nube referida al ámbito de la informática y las de botón referidas a los ámbitos de la música y la botánica, si bien no se encuentra aún registrada ninguna para cintillo a pesar del amplio y extendido uso de esta voz en el ámbito de la publicidad.
} 
labor de traducir los neologismos ${ }^{14}$, si bien, en determinadas ocasiones, para lograr transmitir 'lo mismo' en una lengua y cultura diferentes, pueden crear y aplicar eventualmente estrategias diferentes a las descritas y emplear otras de carácter práctico.

No obstante, aunque existan estrategias teóricas, y también prácticas, que ayudan a resolver el problema de la traducción de los neologismos, muchos traductores optan por transferir directamente -sea vía préstamo sin adaptar, transcripción o transliteración- los nuevos vocablos de la lengua fuente a la lengua de destino sin hacerles ninguna modificación ni adaptación. De ahí que las dificultades se presenten, por tanto, al optar por traducir las nuevas palabras o innovaciones léxicas de la lengua de partida a la lengua de llegada.

Pues bien, nuestras incursiones en la práctica de la traducción, a lenguas orales y signadas, de estas nuevas unidades léxicas de creación reciente y no codificadas lexicográficamente, nos han mostrado (cf. M. T. Díaz Hormigo, 2017b y É. Vega Moreno, 2016c) que, como resultado de esta búsqueda de un equivalente del neologismo de la lengua fuente en la lengua de destino, el traductor puede encontrarse con estas distintas posibilidades: 1) neologismo de lengua fuente se traduce por una unidad léxica registrada en los diccionarios de la lengua meta o de destino ${ }^{15}$; 2) neologismo de lengua fuente se traduce por una unidad léxica no registrada en los diccionarios de la lengua meta pero sí documentada en el uso de la lengua de llegada (= neologismo de la lengua de destino) ${ }^{16}$, y 3) neologismo de lengua fuente se

\footnotetext{
${ }^{14}$ A este respecto, destacamos la propuesta de P. Newmark (1988: 140-150), quien enumera diez estrategias - procedures en su terminología- para la traducción de las nuevas unidades léxicas, dependiendo la elección de una u otra estrategia o de una combinación de estas, del tipo de neologismo que se ha de traducir y de ciertos factores contextuales que envuelven tanto a la creación léxica como al proceso de traducción. No obstante, como suele ser habitual entre los traductólogos que mencionan estrategias para la traducción de neologismos, P. Newmark tampoco atiende para su clasificación a la tipología de la creación léxica vigente en la lengua de llegada.

${ }^{15}$ Ejemplo de neologismo de la lengua fuente que se corresponde con una unidad léxica registrada en los diccionarios de la lengua de destino, unidad que, por tanto, está codificada y normalizada en esa lengua, es la traducción al inglés del verbo español clonar en las acepciones correspondientes a informática y telefonía que se ilustran en el contexto:

"Los jefes del PCC no utilizan móviles con número originales, sino clonados, es decir, replicados de números auténticos de usuarios en el exterior de la prisión, lo que complica el seguimiento de las llamadas" (El País, 19/05/2006).

En efecto, la acepción de clonar de 'generación, mediante copia, de productos como tarjetas o teléfonos móviles' encuentra su equivalente en el inglés to clone, cuyas acepciones de 'make an identical copy of'; 'illegally copy the security codes from (a mobile phone) to one or more others as a way of obtaining free calls' figuran ya recogidas en el Oxford English Oxford English Dictionary.

${ }^{16}$ Ejemplo de neologismo de la lengua fuente que no se corresponde con ninguna unidad léxica registrada en los diccionarios de la lengua de destino pero sí con una unidad léxica que se halla documentada en el uso de esta lengua, por lo que esta unidad léxica, que también es neologismo o innovación léxica en la lengua de destino, sí puede ser usada como equivalente en la traducción, es la traducción a diversas lenguas de la unidad léxica del español micromecenazgo, que aparece ejemplificada en el contexto:
} 
traduce por una unidad léxica no registrada en los diccionarios de lengua $\mathrm{B}$ y no documentada en el uso de la lengua meta o de destino.

En este tercer caso, cuando no existe tal equivalente en la lengua de destino, se presentan como opciones de traducción: a) una paráfrasis en la lengua de destino, o b) la creación ex profeso de un neologismo en la lengua de destino atendiendo a los recursos y tendencias vigentes en esta lengua ${ }^{17}$. De ahí el interés y la conveniencia de realizar estudios contrastivos que determinen los recursos y las tendencias vigentes en las diferentes lenguas para la creación de nuevas unidades léxicas y actualizar su léxico.

\section{NEOLOGÍA Y CoMUNICACIÓN Y ASESORAMIENTO LINGÜÍSTICOS}

En las últimas décadas, han alcanzado especial auge y relevancia los análisis y descripciones de los recursos que se utilizan para la creación léxica en 1) los diferentes tipos de lenguaje (periodístico, publicitario ${ }^{18}$, administrativo, político), para medir la vitalidad de la lengua en estos sectores de la comunicación especializada ${ }^{19}$; 2) las más destacadas esferas de la comunicación social, como la comunicación médico-paciente, administración-ciudadano, empresa-cliente, y 3) los distintos estilos y registros (lenguaje oral / lenguaje escrito) y según las variables sociolingüísticas de sexo, edad y estrato social.

Focalizar la descripción en el hallazgo de la motivación de las nuevas creaciones léxicas, tomando como base la dicotomia creación léxica como necesidad denominativa $v s$. creación léxica como estrategia comunicativa,

\footnotetext{
"Uno de los pioneros en los incipientes orígenes del micromecenazgo fue el grupo británico de rock Marillion, que en 1997 ya financió su gira por EE. UU. gracias a sus fans americanos, que aportaron un total de 60" (Levante, 21/02/2011),

y puede ser traducido al inglés como crowdfunding; francés financement participatif o micromécénat; catalán micromecenatge; portugués financiamento coletivo. Sin embargo, ninguno de estos vocablos aparece registrado en los principales diccionarios de referencia de estas lenguas.

${ }_{17}$ Sirvan como ejemplos de neologismos de la lengua fuente que no se corresponden con ninguna unidad léxica registrada en los diccionarios de la lengua de destino ni con ninguna que se halle documentada en el uso de esta lengua las traducciones a otras lenguas de las denominaciones de seguidores de equipos de fútbol y de tendencias y líderes políticos españoles, como cadista, bético, sevillista; podemitas, pedristas, etc., cuyos equivalentes son, en inglés Cádiz/Sevilla Football Club supporter, Real Betis Balompié supporter; en francés, Partisan du Cádiz football club, partisan du Real Betis Balompié y les partisans de Pedro Sánchez, y. en italiano, il cadista, il bético; I seguaci di Podemos.

${ }^{18} \mathrm{La}$ creación léxica en el lenguaje publicitario ha sido ampliamente estudiada por É. Vega Moreno, fundamentalmente, en sus trabajos de 2010, 2015, 2016a, 2016b, 2017a, 2017b y 2018.

${ }^{19} \mathrm{Y}$ esto es porque estas creaciones léxicas contribuyen a reflejar el estado y la evolución del desarrollo técnico, social y cultural de la sociedad que habla esa lengua, afectando a la calidad y estabilidad de este sistema lingüístico y mostrando, a su vez, la capacidad de este para modificarse y evolucionar a través del tiempo adaptándose a las circunstancias y a las nuevas pretensiones de los hablantes. De ahí que, como se ha indicado en n. 5, uno de los objetivos de los proyectos de investigación sobre la neología y los neologismos sea precisamente contar y valerse de un corpus de neologismos para poder medir la vitalidad de la lengua.
} 
ha redundado en la elaboración de pautas de creación léxica para la consecución de la adecuación y corrección lingüisticas de estos neologismos. En este sentido, se ha de advertir que el grado de aceptabilidad de las nuevas unidades léxicas depende en buena parte, aunque no de manera exclusiva, del nivel de consideración social que alcance un neologismo, si bien, a su vez, se encuentra estrechamente vinculado a la conciencia de necesidad designativa de un concepto, objeto o realidad. Por ello, la cuestión de la aceptabilidad de una creación léxica guarda conexión con la común delimitación entre unidades léxicas creadas para denominar nuevos conceptos, objetos o realidades -neologismos denominativos o referenciales-y unidades léxicas creadas para introducir matices subjetivos o nuevas formas expresivas $\mathrm{u}$ originales en la comunicación -neologismos estilísticos o expresivos-. Pero, a su vez, en cada uno de estos tipos de neología, que surgen de la aplicación del criterio de la función o finalidad de la creación léxica, se pueden, desde nuestro punto de vista, establecer matizaciones y otras subdistinciones, como, entre otras, las fijadas por nuestra parte, para las creaciones léxicas que son creaciones eufemísticas, en M. T. Diaz Hormigo (2012c). En efecto, las creaciones léxicas pueden resultar de una actuación consciente, pues se puede crear una nueva unidad léxica para atraer la atención del destinatario o singularizar el discurso. Asimismo, estas creaciones conscientes y, por tanto, planificadas pueden ser individuales o, lo que es más frecuente, institucionales. En cualquier caso, los neologismos planificados pueden surgir de la necesidad social y política, no solo de denominar un concepto nuevo, sino también de reemplazar una denominación no considerada idónea. Y, para ser rigurosos en el análisis, habria, entre otros aspectos, que especificar la amplia gama de motivaciones extralingüísticas que pueden subyacer a los neologismos estilísticos o expresivos que pretenden sustituir denominaciones no adecuadas. Por ello, la creación léxica es considerada como una estrategia para la consecución de un lenguaje políticamente correcto (corrección política), siendo los más frecuentados ámbitos de actuación el lenguaje no sexista; la enfermedad, las discapacidades, los defectos corporales; los estragos de la edad; el trabajo y la economía (pobreza, emigración, esclavitud, hambre); el racismo y la xenofobia; la guerra y el terrorismo, y el sexo (homosexualidad, transexualidad, pederastia, prostitución) ${ }^{20}$.

\footnotetext{
20 Sirvan como ejemplos unidades léxicas como desaceleración económica por crisis, crecimiento negativo por caída de la producción, recesión por situación económica grave y duradera, regulación de empleo por despido masivo de trabajadores; reajuste, retoque o revisión de tarifas por subida de precios, concurso de acreedores por suspensión de pagos, proliferación de manchas localizadas por marea negra, ataque aliado por guerra, daños colaterales por víctimas civiles, respaldo humanitario por apoyo logístico o apoyo militar, así como deseconomía, reconversión, intervención militar, fuego amigo, comando, lucha armada, conflicto armado, brazo armado, alto el fuego, etc., creadas y utilizadas sobre todo por políticos, pero también por militares, empresarios, economistas, etc., con el único fin o propósito de maquillar, deformar, difuminar o disimular la realidad que pretenden esconder. Frente a estas, las siguientes creaciones del denominado actualmente lenguaje políticamente correcto: larga y penosa enfermedad por cáncer, magrebi por moro, subsahariano o afroamericano por negro, invidente por ciego, discapacitado psíquico por retrasado mental, tercera edad por vejez, muerte voluntaria
} 


\section{Neología, Psicolingüística, Teoría y metodología de enseñanza de Lenguas y Lingǘística Clínica}

Otras aplicaciones de las investigaciones sobre la neología y los neologismos las encontramos en el ámbito de la psicolingüística, pues, tal como quedó demostrado en M. T. Díaz Hormigo y C. Varo Varo (2012), la investigación en neología contribuye a validar los principales modelos de producción e interpretación de unidades léxicas mediante la aplicación de las fases del procesamiento de unidades léxicas conocidas al procesamiento de unidades léxicas nuevas. Asimismo, los resultados de esta aplicación pueden ser incorporados a las teorias y metodologías de la enseñanza de lenguas, pues permiten la formulación de pautas para la enseñanza/aprendizaje y comprensión de estas unidades léxicas recién creadas en la lengua. E, igualmente, en el ámbito de la lingüística clínica, la investigación en neología y sobre los neologismos pueden ser de gran utilidad y aplicabilidad para la detección de determinados trastornos del lenguaje y el tratamiento y rehabilitación de pacientes afectados por determinados tipos de afasias, por ser la anomia y, por tanto, la creación de nuevas unidades léxicas, algunas de las características más acusadas de estas patologías.

\section{Conclusiones}

En este artículo hemos dado cuenta de la interrelación e interdependencia de la neología y determinadas disciplinas de la lingüística aplicada. En concreto, hemos referido, sobre todo, las conexiones entre la neología y la lexicografia, la política y planificación lingüisticas, la gramática, la terminología, la traductología y la comunicación y el asesoramiento lingüísticos, pero también, aunque de manera más somera, entre la neología y la psicolingüística, la teoría y metodología de la enseñanza de lenguas, y la lingüística clínica. Por ello, podemos aseverar la existencia de transferencia de los resultados de la investigación en neología y sobre las unidades léxicas recientemente creadas y no codificadas lexicográficamente a otros sectores y el aprovechamiento en neología de los avances en estas disciplinas, campos de actuación o aplicabilidades de la lingüística. No obstante, insistimos en la conveniencia de continuar indagando sobre las aplicabilidades aquí señaladas y detectar y emprender nuevas vias de transferencias hacia otros campos de la lingüística aplicada aquí no referidos.

por suicidio se insertan en determinados ámbitos y dominios interdictivos de carácter sociopolítico, como, fundamentalmente, las enfermedades, discapacidades, defectos corporales, estragos de la edad, racismo, xenofobia, sexo, etc. para minimizar las ofensas a los grupos afectados. 


\section{REFERENCIAS}

CABRÉ, M. T. (1993): La terminología. Teoria, metodología, aplicaciones, Barcelona: Editorial Antártida / Empúries.

CABRÉ, M. T. et al. (2002): "Evaluación de la vitalidad de una lengua a través de la neología: a propósito de la neología espontánea y de la neología planificada", Cabré, M. T. et al. (eds.): Lèxic i neologia, Barcelona: Universitat Pompeu Fabra, Institut Universitari de Lingüística Aplicada, Observatori de Neologia, pp. 159-201.

CABRÉ, M. T. et al. (2004): Metodología del trabajo en neologia: criterios, materiales y procesos, Barcelona: IULA. Publicación electrónica:

http:/ /hdl.handle.net/2072/8

5 (Fecha de consulta: 15/01/2018).

DÍAZ HORMIGO, M. T. (2008). "La investigación lingüística de la neología léxica en España. Estado de la cuestión", LynX. Panorámica de estudios lingüisticos, 7, pp. 5-60.

DÍAZ HORMIGO, M. T. (2011a): “Word Formation Processes and Proposals for the Classification of Formal Neologisms", Cifuentes Honrubia, J. L. y Rodríguez Rosique, S. (eds.): Spanish Word Formation and Lexical Creation, Amsterdam/Philadelphia: John Benjamins, pp. 347-367.

DÍAZ HORMIGO, M. T. (2011b): "Sobre los denominados sustantivos deverbales de acción", Lorenzo Hervás. Documentos de trabajo de Lingüistica Teórica y General. Homenaje a Valerio Báez San José, 20, pp. 123174. Publicación electrónica: earchivo.uc3m.es/bitstream/100
16/11540/5/Lorenzo_Hervas_20.pdf (Fecha de consulta: 28/03/2018).

DÍAZ HORMIGO, M. T. (2012a): "La formación de palabras: tendencias predominantes en la derivación", Montoro del Arco, E. T. (ed.): Neologia y creatividad lingüistica. Quaderns de Filologia de la Universitat de València, 77, pp. 83-112.

DÍAZ HORMIGO, M. T. (2012b): "Consideraciones sobre la flexión de los compuestos sintagmáticos", Fábregas, A. et al. (eds.): Los limites de la morfología. Estudios ofrecidos a Soledad Varela Ortega, Madrid: Ediciones de la Universidad Autónoma de Madrid, pp. 133-143.

DÍAZ HORMIGO, M. T. (2012c): "Lexical Creation and Euphemism: Regarding the Distinction Denominative or Referential Neology vs. Stylistic or Expressive Neology", Lexis. Journal in English Lexicology, 7, pp. 107120. Publicación electrónica: https://journals.openedition.org/lexis/371 (Fecha de consulta: 28/04/2018).

DÍAZ HORMIGO, M. T. (2015): "Neología aplicada y lexicografia: para la (necesaria) actualización de las entradas de los elementos de formación de palabras en diccionarios generales", Revista de Lingüistica y Lenguas Aplicadas, 10, pp. 12-20. Publicación electrónica: https://polipapers.upv.es/index.php/rdlyla/article/view/3587 (Fecha de consulta: 25/09/2018). DOI: https:/ / doi.org/10.4995/rlyla. 2015.3587.

DÍAZ HORMIGO, M. T. (2017a): "Neología y niveles lingüísticos: a 
propósito del análisis morfológico de las creaciones léxicas", Almeida Cabrejas, B. et al. (eds.): Investigaciones actuales en Lingüistica. Vol. II. Semántica, Lexicología y Morfologia, Alcalá de Henares: Universidad de Alcalá, pp. 51-65.

DÍAZ HORMIGO, M. T. (2017b): "Reflections on the translation of neological lexical units", Bécart, A. et al. (eds.): Current Approaches to Translation and Interpretation Studies, Sevilla: Editorial Bienza, pp. 13-18.

DÍAZ HORMIGO, M. T. \& VARO VARO, C. (2012): "Neology and cognition", Eizaga, B. (ed.): Studies in Linguistics and Cognition, Frankfurt am Main: Peter Lang, pp. 15-34.

DÍAZ HORMIGO, M. T.; VARO VARO, C. \& VEGA MORENO, É. (2016): "Las investigaciones del Nodo de Neología de la Universidad de Cádiz (NEOUCA)", Sánchez Manzanares, M. C. (ed.): Estudios de neología del español, Murcia: Editum, pp. 79-107.

NEWMARK, P. (1988): A textbook of Translation, New York/London/Toronto/Sydney/Tokyo: Prentice Hall.

REAL ACADEMIA ESPAÑOLA (2014, $23^{a}$ ed.): Diccionario de la lengua española, Madrid: Espasa.

RONDEAU, G. (1984): Introduction à la terminologie, (Québec): Gaëtan Morin.

SÁNCHEZ MANZANARES, C. (dir.); AZORÍN FERNÁNDEZ， D. \& SANTAMARÍA PÉREZ, I. (2016): NEOMA. Diccionario de neologismos del español actual, Murcia: Editum.

VEGA MORENO, É. (2010): Las creaciones neológicas en el lenguaje de la publicidad, Cádiz: Universidad de Cádiz. Trabajo de in- vestigación inédito para la obtención del Diploma de Estudios Avanzados.

VEGA MORENO, É. (2015): "La creación neológica como herramienta publicitaria", González Sanz, M. (coord.): Jóvenes aportaciones a la investigación lingüistica, Sevilla: Ediciones Alfar, pp. 351-365.

VEGA MORENO, É. (2016a): La creatividad léxica en el lenguaje de la publicidad. Análisis lingüistico de los mecanismos de producción e interpretación neológicas en los sectores de la estética y la peluquería, Cádiz: Universidad de Cádiz. Tesis doctoral inédita.

VEGA MORENO, É. (2016b): "Bases metodológicas para el estudio de las creaciones léxicas en el lenguaje de la publicidad", Ruano San Segundo, P. et al. (eds.): Nuevos enfoques en investigación lingüística, Cáceres: Universidad de Extremadura, pp. 57-73.

VEGA MORENO, É. (2016c): "El préstamo como procedimiento de creación neológica", Pragmalingüistica, 24, pp. 281-292.

VEGA MORENO, É. (2017a): "La creatividad léxica en el lenguaje de la publicidad. Análisis lingüístico de los mecanismos de producción e interpretación neológicas en los sectores de la estética y la peluquería”, Fernández Gómez, S. et al. (eds.): Investigaciones actuales en Lingüistica. Vol. VII. Planes de investigación de doctorado, Alcalá de Henares: Universidad de Alcalá, pp. 63-77.

VEGA MORENO, É. (2017b): "Productividad de los mecanismos de creación léxica en el lenguaje de la publicidad", Olza, I. et al. 
(eds.): Semántica, léxico y fraseologia, Frankfurt am Main: Peter Lang, pp. 145-160.

VEGA MORENO, É. (2017c): "Las fichas neológicas como herramientas de investigación lingüistica", Lingüística en la Red, XV. Publicación electrónica: http://www.linred.es/numero14.html, pp. 1-14 (Fecha de consulta: 20/05/2018).

VEGA MORENO, É. (2018): "Acercamiento al estudio de las unidades neológicas en el sector publicitario de la alimentación", Diaz, M. et al. (eds.): Actas do XIII Congreso Internacional de Lingüistica Xeral. 13-15 de xuño de 2018, Vigo: Universidade de Vigo, pp. 912-919.

VEGA MORENO, É. (en prensa). "Nuevos enfoques metodológicos para el estudio de las creaciones léxicas", Hernández Dias, A. y Pérez Baraja, A. E. (eds.): Propuestas metodológicas para la investigación lingüística, Colima: Universidad de Colima.

VEGA MORENO, É. \& LLOPART SAUMELL, E. (2017): "Delimitación de los conceptos de 'novedad' y 'neologicidad"', Rilce: Revista de Filología Hispánica, 33.3: 14161451. 